\begin{tabular}{|c|c|}
\hline $\begin{array}{c}\text { PRAMANA } \\
\begin{array}{c}\text { journal of } \\
\text { physics }\end{array}\end{array}$ & (c) Indian Academy of Sciences \\
\hline
\end{tabular}

\title{
Quasi-particle properties in a quasi-two-dimensional electron liquid
}

\author{
R ASGARI $^{1, *}$ and B TANATAR ${ }^{2}$ \\ ${ }^{1}$ School of Physics, Institute for Studies in Theoretical Physics and Mathematics, \\ 19395-5531 Tehran, Iran \\ ${ }^{2}$ Department of Physics, Bilkent University, Bilkent, 06800 Ankara, Turkey \\ ${ }^{*}$ Corresponding author. E-mail: asgari@theory.ipm.ac.ir
}

\begin{abstract}
We consider the quasi-particle properties such as the effective mass and spin susceptibility of quasi-two-dimensional electron systems. The finite quantum well width effects are incorporated into the local-field factors that describe the charge and spin correlations. We employ the Fermi-hypernetted chain formalism in conjunction with fluctuationdissipation theorem to obtain the local-field factors. Our results are in good agreement with recent experiments.
\end{abstract}

Keywords. 2D electron liquid; effective mass; spin susceptibility.

PACS Nos 71.10.Ca; 73.20.Mf; 73.40.-c

\section{Introduction}

Two-dimensional (2D) electron systems occurring in semiconductor interfaces are of continuing interest $[1,2]$ both from basic physics and technological points of view. A wealth of theoretical and experimental activity came about to understand the metal-insulator (MIT) observed in Si-MOSFET- and GaAs-based structures [3]. The experiments [4-14] are performed on low-density samples where the interaction effects are important and it is becoming clear that to understand the observed behavior, realistic modeling of the sample geometry is very important. As the systems have an extension in the perpendicular direction, they are quasi-two-dimensional (Q2D) and this feature needs to be taken into account in theoretical calculations.

Experiments [6,7] of in-plane magnetoresistance in Si inversion layers suggested a ferromagnetic instability close to the critical density for the Q2D MIT driven by a divergence in the effective mass. Pudalov et al [8] measured the effective mass and spin susceptibility in the vicinity of the Q2D MIT, finding no evidence for a divergent behavior but a moderate enhancement of the effective mass by a factor of $\approx 2-$ 2.5 over the band mass. Two groups have also reported anomalous density dependence of the modified Landé factor in $n$-doped [9] and $p$-doped [10] GaAs/AlGaAs heterojunctions that are in disagreement with results in Si-MOSFETs. The electron density dependence of the spin susceptibility has been studied by Zhu et al [12] who used high quality Q2D samples. More recently, Tan et al [11] performed 
measurements of the electronic effective mass in Q2D electron system over a wide range of electron density. Spin polarization for a Q2D electron system has been studied by a combination of measurements and calculations by Tutuc et al [13]. Their results revealed the importance of finite thickness of the electron layer and the resulting deformation of the energy surface in the presence of a parallel magnetic field induces an enhancement of the effective mass and Landé $g^{*}$-factor.

Theoretical calculations of the effective mass and spin susceptibility of electron systems are performed within the framework of Fermi liquid theory, the key ingredient of which is the quasi-particle concept and its interactions. As applied to the electron gas model this entails the calculation of effective electron-electron interactions which enter the many-body formalism allowing the calculation of various physical properties. A number of calculations considered the self-energy [15-19] from which density, spin polarization, and temperature dependence of effective mass are obtained. In these calculations the on-shell approximation $[16,17]$ yields a diverging effective mass but the full solution of Dyson equation yields only a mild enhancement $[18,19]$.

In a recent paper De Palo et al [20] employed quantum Monte Carlo simulation results for a $2 \mathrm{D}$ electron system in conjunction with perturbation theory using the parameters of specific samples of Zhu et al [12] to calculate the spin susceptibility and stressed the importance of Q2D nature of the physical systems. Dharmawardana in a series of papers [21] has calculated the effective mass, Landé $g^{*}$-factor and spin susceptibility for Q2D electron systems within the classical-map hypernetted chain (CHNC) approximation. He found that the thickness effect on the spin-phase transition provides a clear picture of the changes in the spin susceptibility enhancement leading to a strong increase in the $g^{*}$-factor, while the effective mass is increased from the reduction of the Coulomb potential in thick layers.

In this paper we study the quasi-particle properties such as effective mass and spin susceptibility of Q2D electron systems in the density range relevant to recent experiments of Tan et al [11] and Zhu et al [12]. Previously [19] we studied a strictly $2 \mathrm{D}$ electron system and calculated the effects of correlations and disorder in the effective mass enhancement. More recently, we concentrated [18] on Q2D systems, but we had employed local-field factors which were built from the quantum Monte Carlo (QMC) data and were valid for strictly 2D systems. Local-field factors embody correlation effects beyond the random phase approximation (RPA) and constitute a significant input to our calculations at intermediate couplings. To this end, we use static structure factors resulting from a Fermi-hypernetted chain calculation (FHNC) [22-25] in conjunction with the fluctuation-dissipation theorem to extract the local-field factors which depend on the quantum-well width. For the specific sample parameters of Tan et al [11] experiments, we find good agreement with the observed spin susceptibility. Our results are also in good agreement with the QMC simulations of De Palo et al [20] in the same range of coupling strengths.

\section{Theory}

We consider a Q2D electron gas with band mass $m$ in a semiconductor heterostructure with dielectric constant $\kappa$. We include the effect of thickness of a GaAs heterojunction into the bare electron-electron interaction $v_{\mathbf{q}}=2 \pi e^{2} F(q d) /(\kappa q)$ 
which is the Coulomb potential renormalized by the form factor given by

$$
F(x)=\left(1+\frac{\kappa_{\mathrm{ins}}}{\kappa_{\mathrm{sc}}}\right) \frac{8+9 x+3 x^{2}}{16(1+x)^{3}}+\left(1-\frac{\kappa_{\mathrm{ins}}}{\kappa_{\mathrm{sc}}}\right) \frac{1}{2(1+x)^{6}},
$$

where $d=\left[\hbar \kappa_{\mathrm{sc}} /\left(48 \pi m e^{2} n^{*}\right)\right]^{1 / 3}$ and $n^{*}=n_{\mathrm{depl}}+11 n / 32$. Here the depletion layer charge density $n_{\text {depl }}$ is essentially zero and $\kappa_{\text {ins }}=10.9$ and $\kappa_{\text {sc }}=12.9$ and $\kappa$ is their average. At zero temperature there are only two parameters for a homogeneous Q2D electron gas, the Wigner-Seitz parameter $r_{\mathrm{s}}=\left(\pi n a_{\mathrm{B}}^{2}\right)^{-1 / 2}$, where $a_{\mathrm{B}}=\hbar^{2} \kappa /\left(m e^{2}\right)$ is the effective Bohr radius, and the degree of spin polarization $\zeta=\left|n_{\uparrow}-n_{\downarrow}\right| / n$. Here $n_{\sigma}$ is the average density of particles with spin $\sigma=\uparrow, \downarrow$ and $n=n_{\uparrow}+n_{\downarrow}$ is the total average density. We also define the Fermi energy and wave number by $\varepsilon_{\mathrm{F}}=\hbar^{2} k_{\mathrm{F}}^{2} /(2 m)$ and $k_{\mathrm{F}}=\left(2 \pi n_{2 \mathrm{D}}\right)^{1 / 2}=\sqrt{2} /\left(r_{\mathrm{s}} a_{\mathrm{B}}\right)$, respectively.

To calculate the quasi-particle $(\mathrm{QP})$ properties, we start with the calculation of the retarded self-energy, which can be decomposed in the usual way into the frequency-independent Hartree-Fock and frequency-dependent correlation parts $[15,17]$. The correlation part of the self-energy involves the effective QP interaction between the electrons for which we use the Kukkonen-Overhauser form [15,26]. The main ingredient of this formalism is the screening dielectric function

$$
\frac{1}{\varepsilon(\mathbf{q}, \omega)}=1+v_{\mathbf{q}}\left[1-G_{+}(\mathbf{q})\right]^{2} \chi_{\mathrm{C}}(\mathbf{q}, \omega)+3 v_{\mathbf{q}} G_{-}^{2}(\mathbf{q}) \chi_{\mathrm{S}}(\mathbf{q}, \omega) \text {. }
$$

In this expression $\chi_{\mathrm{C}}(\mathbf{q}, \omega)$ and $\chi_{\mathrm{S}}(\mathbf{q}, \omega)$ represent the charge-charge and spin-spin response functions, which in turn are defined and determined by the spin-symmetric and spin-antisymmetric local field factors $G_{+}(\mathbf{q})$ and $G_{-}(\mathbf{q})$ via the relations

$$
\chi_{\mathrm{C}, \mathrm{S}}(\mathbf{q}, \omega)=\frac{\chi_{0}(\mathbf{q}, \omega)}{1-f_{\mathrm{C}, \mathrm{S}}(\mathbf{q}) \chi_{0}(\mathbf{q}, \omega)},
$$

where $f_{\mathrm{C}}(\mathbf{q})=v_{\mathbf{q}}\left[1-G_{+}(\mathbf{q})\right], f_{\mathrm{S}}(\mathbf{q})=-v_{\mathbf{q}} G_{-}(\mathbf{q})$ and $\chi_{0}(\mathbf{q}, \omega)$ is the response function of a noninteracting system. In the paramagnetic electron liquid $G_{ \pm}(\mathbf{q})=\left[G_{\uparrow \uparrow}(\mathbf{q}) \pm G_{\uparrow \downarrow}(\mathbf{q})\right] / 2$, where $G_{\sigma \sigma^{\prime}}(\mathbf{q})$ are the spin-resolved local-field factors. Note that we have approximated the local-field factors by their static, frequency-independent limits.

Quite generally, once the QP self-energy is known, the QP excitation energy $\delta \mathcal{E}_{\mathrm{QP}}(\mathbf{k})$, which is the QP energy measured from the chemical potential $\mu$ of the interacting system, can be calculated by solving self-consistently the Dyson equation

$$
\delta \mathcal{E}_{\mathrm{QP}}(\mathbf{k})=\xi_{\mathbf{k}}+\left.\Re \Sigma_{\text {ret }}^{\mathrm{R}}(\mathbf{k}, \omega)\right|_{\omega=\delta \mathcal{E}_{\mathrm{QP}}(\mathbf{k}) / \hbar},
$$

where $\Re \Sigma_{\text {ret }}^{\mathrm{R}}(\mathbf{k}, \omega)=\Re \Sigma_{\text {ret }}(\mathbf{k}, \omega)-\Sigma_{\text {ret }}\left(k_{\mathrm{F}}, 0\right)$. For later purposes we introduce at this point the so-called on-shell approximation (OSA). This amounts to approximating the QP excitation energy by calculating $\Re \Sigma_{\text {ret }}^{\mathrm{R}}(\mathbf{k}, \omega)$ in eq. (4) at the frequency $\omega=\xi_{\mathbf{k}} / \hbar$.

Once the QP excitation energy is known, the effective mass $m^{*}(k)$ can be calculated by means of the relationship

$$
\frac{1}{m^{*}(k)}=\frac{1}{\hbar^{2} k} \frac{\mathrm{d} \delta \mathcal{E}_{\mathrm{QP}}(k)}{\mathrm{d} k}
$$


Evaluating $m^{*}(k)$ at $k=k_{\mathrm{F}}$, one gets the QP effective mass at the Fermi surface. We remark that the QP excitation energy may be calculated either by solving self-consistently the Dyson equation or using the OSA.

Starting with the quasi-particle energy and its relation to the Landau interaction function, one can derive the modified Landé $g^{*}$-factor expression [15].

$$
\frac{g}{g^{*}}=1+\frac{m^{*}}{2 \pi} \int_{0}^{2 \pi} \frac{\mathrm{d} \phi}{2 \pi}\left(\frac{\delta^{2} E}{\delta n_{\mathbf{k}}^{\uparrow} \delta n_{\mathbf{p}}^{\uparrow}}-\frac{\delta^{2} E}{\delta n_{\mathbf{k}}^{\uparrow} \delta n_{\mathbf{p}}^{\downarrow}}\right),
$$

where $E$ is the total ground state energy. Here $|\mathbf{k}|=|\mathbf{P}|=\mathbf{k}_{\mathrm{F}}$ and $\phi$ is the angle between them. Once the QP effective mass $m^{*}$ and modified Landé $g^{*}$-factor have been calculated, the spin susceptibility is found by the following exact relationship:

$$
\frac{\chi^{*}}{\chi_{0}}=\frac{m^{*}}{m} \frac{g^{*}}{g},
$$

where $\chi_{0}$ is the Pauli spin susceptibility.

As is clear from eqs (2) and (3) the local-field factors are the fundamental quantities for an evaluation of quasi-particle properties. Our strategy is to use accurate spin-symmetric and spin-antisymmetric static structure factors to build the local-field factors [27]. For this purpose we use the Fermi hypernetted-chain approach [22-25] to calculate the spin-symmetric and spin-antisymmetric static structure factors incorporating the finite thickness effects in a quantum well.

Within the FHNC approach, a formally exact differential equation for the paircorrelation function $g_{\sigma \sigma^{\prime}}(r)$ reads

$$
\left[-\frac{\hbar^{2}}{m} \nabla_{\mathbf{r}}^{2}+v(r)+v_{\mathrm{P}}^{\sigma \sigma^{\prime}}(r)+V_{\mathrm{EKS}}^{\sigma \sigma^{\prime}}(r)\right] \sqrt{g_{\sigma \sigma^{\prime}}(r)}=0 .
$$

Here, $v(r)$ is the Q2D potential and the 'Pauli potential' $v_{\mathrm{P}}^{\sigma \sigma^{\prime}}(r)$ is defined by [28]

$$
v_{\mathrm{P}}^{\sigma \sigma^{\prime}}(r)=\frac{\hbar^{2}}{m} \frac{\nabla_{\mathbf{r}}^{2} \sqrt{g_{\sigma \sigma^{\prime}}^{\mathrm{HF}}(r)}}{\sqrt{g_{\sigma \sigma^{\prime}}^{\mathrm{HF}}(r)}} .
$$

Although the expression for the Pauli potential is exact only for a weakly coupled $2 \mathrm{D}$ electron gas [28], we shall assume in the following that it can provide useful results in our FHNC approach in Q2D. The FHNC expresses the potential $V_{\mathrm{EKS}}^{\sigma \sigma^{\prime}}(r)$ in eq. (8), which is the sum of the Hartree and of the exchange-correlation potential, as the sum of two effective pair interactions [22-24]:

$$
V_{\mathrm{EKS}}^{\sigma \sigma^{\prime}}(r)=W_{\mathrm{B}}^{\sigma \sigma^{\prime}}(r)+\delta_{\sigma \sigma^{\prime}} W_{\mathrm{e}}^{\sigma \sigma}(r) .
$$

We introduce the partial structure factors $S_{\sigma \sigma^{\prime}}(q)$ of the binary mixture which are the Fourier transforms of $g_{\sigma \sigma^{\prime}}(r)$. We also introduce the Fourier transform of $W_{\mathrm{B}}^{\sigma \sigma^{\prime}}(r)\left(W_{\mathrm{B}}^{\sigma \sigma^{\prime}}(q)\right.$, say). Minimization of the ground state energy against arbitrary variations of $g_{\sigma \sigma^{\prime}}(r)$ yields the expression

$$
W_{\mathrm{B}}^{\sigma \sigma^{\prime}}(q)=-\frac{\varepsilon_{q}}{\sqrt{n_{\sigma} n_{\sigma^{\prime}}}}\left[S_{\sigma \sigma^{\prime}}(q)-\delta_{\sigma \sigma^{\prime}}\right]-V_{\sigma \sigma^{\prime}}(q),
$$


where $\varepsilon_{q}=\hbar^{2} q^{2} /(2 m)$ is the single-particle energy and the function $V_{\sigma \sigma^{\prime}}(q)$ is the Fourier transform of the 'particle-hole' interaction. $V_{\sigma \sigma^{\prime}}(r)$ is given by

$$
\begin{aligned}
V_{\sigma \sigma^{\prime}}(r)= & g_{\sigma \sigma^{\prime}}(r)\left[v(r)+W_{\mathrm{e}}^{\sigma \sigma^{\prime}}(r)+v_{\mathrm{P}}^{\sigma \sigma^{\prime}}(r)\right]+\left[g_{\sigma \sigma^{\prime}}(r)-1\right] W_{\mathrm{B}}^{\sigma \sigma^{\prime}}(r) \\
& +\frac{\hbar^{2}}{m}\left|\nabla \sqrt{g_{\sigma \sigma^{\prime}}(r)}\right|^{2} .
\end{aligned}
$$

Turning to the second term on the left-hand side of eq. (10), the effective pair potential $W_{\mathrm{e}}^{\sigma \sigma}(r)$ has a rather complicated expression within the FHNC [22-24]. However, in dealing with a one-component electron fluid, Kallio and Piilo [29] have proposed a simple and effective way to account for this consequence of the antisymmetry of the fermion wave function. Their argument generalized to our twocomponent Fermi fluid leads to the requirement that in Fourier transform this term should cancel the effective boson-like interaction $W_{\mathrm{B}}^{\sigma \sigma}(q)$ for parallel-spin electrons at low coupling. That is,

$$
W_{\mathrm{e}}^{\sigma \sigma}(q)=-\lim _{r_{s} \rightarrow 0} W_{\mathrm{B}}^{\sigma \sigma}(q)=\frac{\varepsilon_{q}}{2 n_{\sigma}}\left[1+2 S_{\sigma \sigma}^{\mathrm{HF}}(q)\right]\left[\frac{S_{\sigma \sigma}^{\mathrm{HF}}(q)-1}{S_{\sigma \sigma}^{\mathrm{HF}}(q)}\right]^{2},
$$

where $S_{\sigma \sigma}^{\mathrm{HF}}(q)$ is the Hartree-Fock structure factor. The above formal set of equations within the FHNC approach are solved self-consistently.

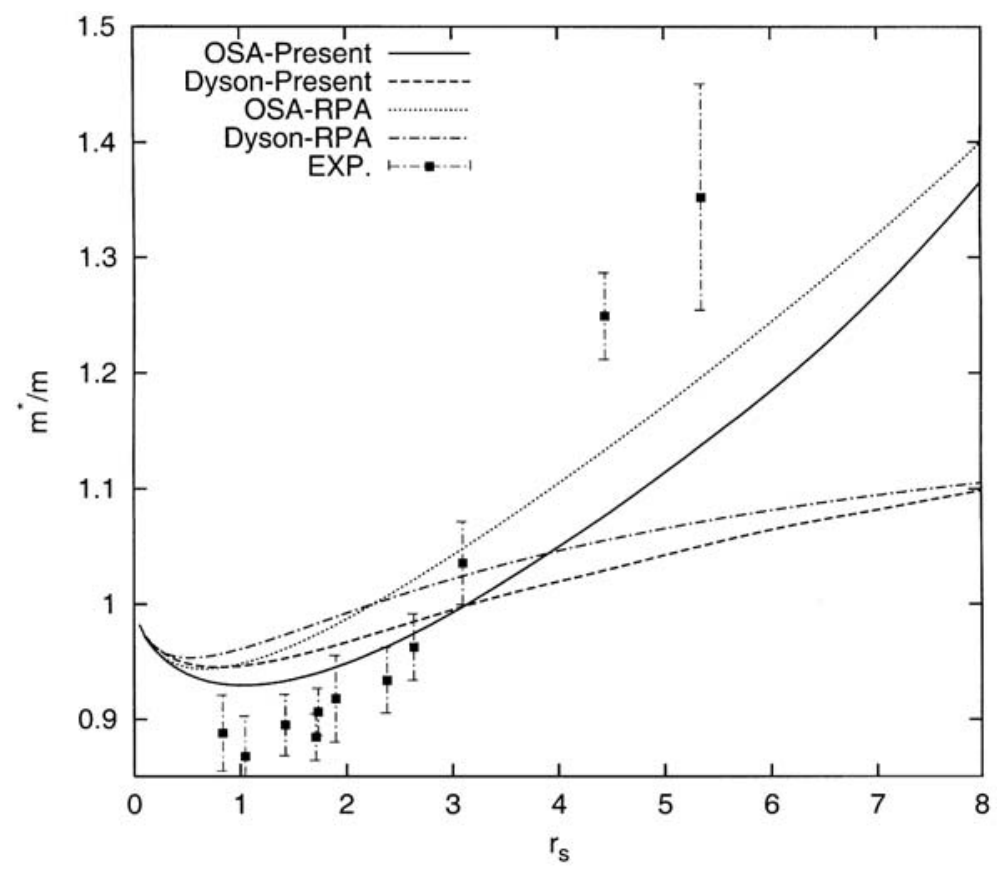

Figure 1. Many-body effective mass as a function of $r_{\mathrm{s}}$ for $0 \leq r_{\mathrm{s}} \leq 8$ for a Q2D electron gas confined in a GaAs/AlGaAs triangular quantum well of the type used in ref. [11]. 


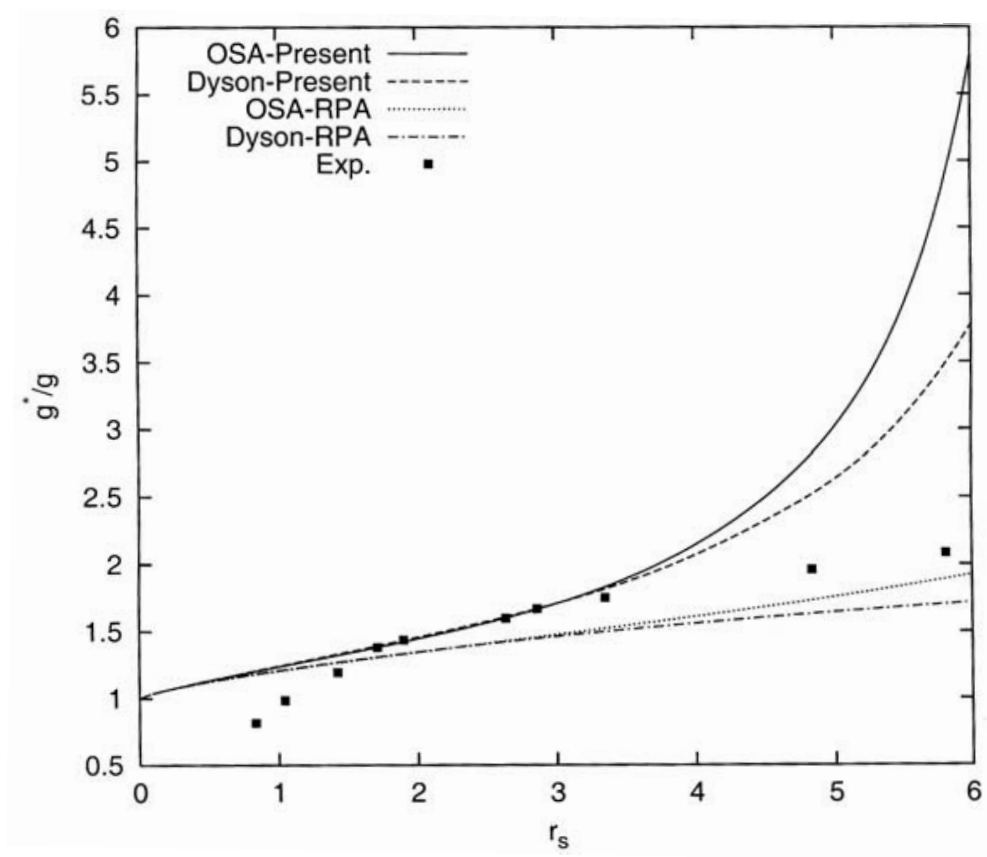

Figure 2. $g^{*} / g$ as a function of $r_{\mathrm{s}}$ for $0 \leq r_{\mathrm{s}} \leq 6$. The experimental data $\chi^{*} m / \chi_{0} m^{*}$ is from the $\chi^{*} / \chi_{0}$ of empirical formula given by ref. [12] divided by the $m^{*} / m$ of ref. [11].

The fluctuation-dissipation theorem relates the dynamic susceptibilities defined above to the static structure factors

$$
S_{ \pm}(q)=-\frac{1}{n \pi} \int_{0}^{\infty} \mathrm{d} \omega \Im\left[\chi_{\mathrm{C}, \mathrm{S}}(q, \omega)\right]
$$

where $S_{ \pm}(q)=\left[S_{\uparrow \uparrow} \pm S_{\uparrow \downarrow}\right] / 2$. As $\chi_{\mathrm{C}}(q, \omega)$ and $\chi_{\mathrm{S}}(q, \omega)$ depend on $G_{+}(q)$ and $G_{-}(q)$, respectively, the above integral expression allows one to determine the local-field factors once the static structure factors are calculated by the FHNC approach.

\section{Results and discussion}

In figure 1 we show our numerical results of the QP effective mass both in OSA and Dyson approximations. The QP effective mass enhancement is substantially smaller in the Dyson equation calculation than in the OSA, because of cancellations in the expression for the Dyson approach $[18,30]$. To clarify the effect of chargeand spin-density fluctuations we have also included the RPA results which do not take the spin fluctuations into account. Comparing the results of figure 1 with the experimental measurements of Tan et al [11] we can draw the following conclusions: (i) RPA and present results are rather similar in the weak coupling limit $\left(r_{\mathrm{s}} \ll\right.$ 1), (ii) theoretical calculations in the strong coupling region are not so close to experimental data. We note that experimental data were collected at weak magnetic 


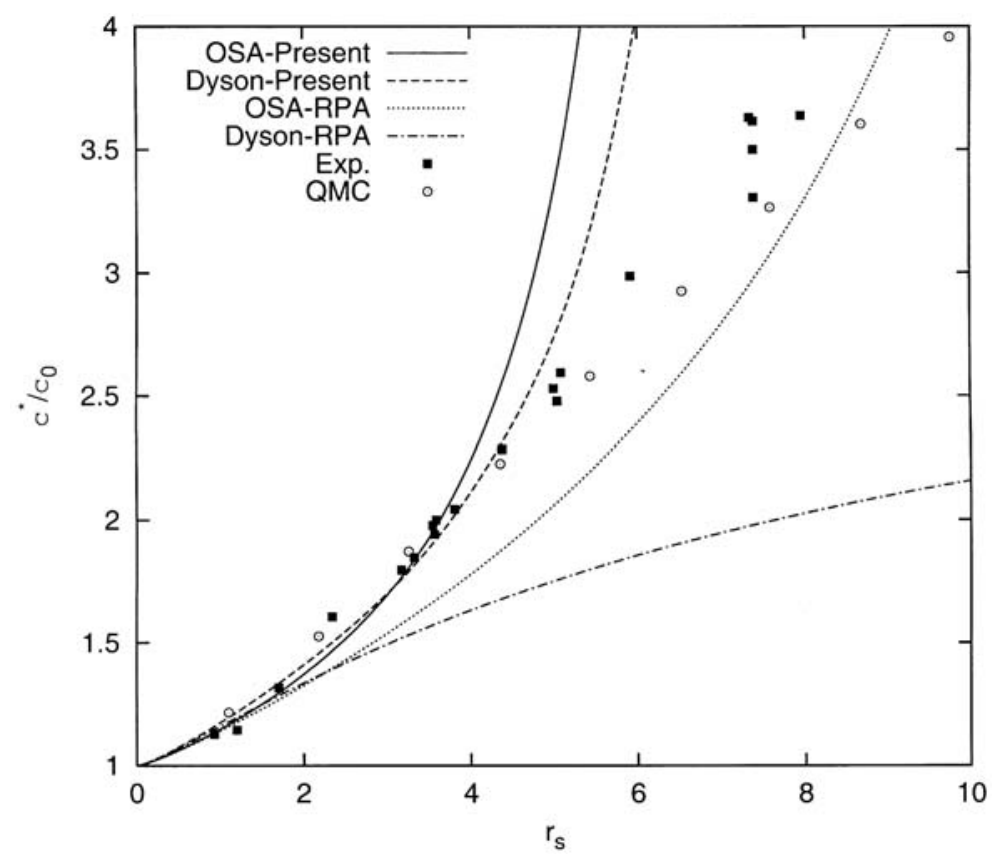

Figure 3. Spin susceptibility as a function of $r_{\mathrm{s}}$ for $0 \leq r_{\mathrm{s}} \leq 10$ for a Q2D electron gas confined in a GaAs/AlGaAs triangular quantum well of the type used in ref. [12] compared with quantum Monte Carlo results of ref. [20].

fields and mostly in high Landau levels. However, our numerical calculations have been performed in the absence of a magnetic field.

Figure 2 displays our results for the ratio $g^{*} / g$ as a function of $r_{\mathrm{s}}$ for $0 \leq r_{\mathrm{s}} \leq 6$. $g^{*} / g$ embodies the charge and spin fluctuation effects through $G_{+}$and $G_{-}$. We included the value of experimental $\chi^{*} m / \chi_{0} m^{*}$ which is extracted from the $\chi^{*} / \chi_{0}$ empirical formula given by Tan et al [12] divided by the experimental data of $m^{*} / m$ of Tan et al [11]. We observe that there is an enhancement in $g^{*}$ beyond $r_{\mathrm{s}} \sim 5$ within the present method using either OSA or the Dyson approaches compared to the experimental data and the RPA calculation. In particular, it is surprising that RPA yields a reasonable agreement with experiment in a region of $r_{\mathrm{s}}$ values where it is not expected to be very reliable.

In figure 3 we show the spin susceptibility as a function of $r_{\mathrm{s}}$ compared to RPA, recent experimental data of Zhu et al [12] and quantum Monte Carlo calculation [20]. As is clear from this figure, $\chi^{*} / \chi$ starts at unity when $r_{\mathrm{s}}$ tends to zero and increases with increasing $r_{\mathrm{s}}$ values. Our numerical calculations within both OSA and Dyson approximations are in good agreement with the experimental measurements in the weak and intermediate coupling limits. Furthermore, the level of agreement we obtain is slightly better than QMC simulations [20,31] which do not take the finite thickness effects into account.

The above results for the spin susceptibility basically reflect the present status of the perturbation theory-based calculations despite the fact that a highly 
sophisticated approach was taken to improve the quasi-particle interactions. The poor agreement with experimental data beyond $r_{\mathrm{s}} \sim 4$ can be considered to be a shortcoming of the formalism. Our calculations indicate that finite thickness effects alone cannot account for the discrepancy. It would be important to improve upon this outstanding theoretical problem. The recent QMC calculation by De Palo et al [20] which represents the experiments quite well, on the other hand, is based on the accurate evaluation of the ground-state energy and therefore is of a different nature than our approach. It is of theoretical interest to bring the level of agreement between different approaches closer.

In conclusion, we have performed the calculation of effective mass and spin susceptibility for a Q2D electron system to compare with recent experiments. The correlation effects are described by local-field factors obtained from self-consistent FHNC formalism. The finite width effects are consistently included in our calculations which improve the agreement with experiments.

\section{Acknowledgment}

This work is supported by TUBITAK (106T052) and TUBA.

\section{References}

[1] T Ando, A B Fowler and F Stern, Rev. Mod. Phys. 54, 437 (1982)

[2] G F Giuliani and G Vignale, Quantum theory of the electron liquid (Cambridge University Press, Cambridge, England, 2005)

[3] E Abrahams, S V Kravchenko and M P Sarachik, Rev. Mod. Phys. 73, 251 (2001)

S V Kravchenko and M P Sarachik, Rep. Prog. Phys. 67, 1 (2004)

[4] V T Dolgopolov, G V Kravchenko, A A Shashkin and S V Kravchenko, JEPT Lett. 55, $733(1992)$

D Simonian, S V Kravchenko, M P Sarachik and V M Pudalov, Phys. Rev. Lett. 79, 2304 (1997)

V M Pudalov, G Brunthaler, A Prinz and G Bauer, JEPT Lett. 65, 932 (1997)

[5] T Okamoto, K Hosoya, S Kawaji and A Yagi, Phys. Rev. Lett. 82, 3875 (1999)

[6] S A Vitkalov, H Zheng, K M Mertes, M P Sarachik and T M Klapwijk, Phys. Rev. Lett. 87, 086401 (2001)

[7] A A Shashkin, S V Kravchenko, V T Dolgopolov and T M Klapwijk, Phys. Rev. Lett. 87, 086801 (2001); Phys. Rev. B66, 073303 (2002)

[8] V M Pudalov, M E Gershenson, H Kojima, N Butch, E M Dizhur, G Brunthaler, A Prinz and G Bauer, Phys. Rev. Lett. 88, 196404 (2002)

[9] E Tutuc, S Melinte and M Shayegan, Phys. Rev. Lett. 88, 036805 (2002)

[10] H Noh, M P Lilly, D C Tsui, J A Simmons, E H Hwang, S Das Sarma, L N Pfeiffer and K W West, Phys. Rev. B68, 165308 (2003)

[11] Y-W Tan, J Zhu, H L Stormer, L N Pfeiffer, K W Baldwin and K W West, Phys. Rev. Lett. 94, 016405 (2005)

[12] J Zhu, H L Stormer, L N Pfeiffer, K W Baldwin and K W West, Phys. Rev. Lett. 90, 056805 (2003)

Y-W Tan, J Zhu, H L Stormer, L N Pfeiffer, K W Baldwin and K W West, Phys. Rev. B73, 045334 (2006) 
[13] E Tutuc, S Melinte, E P De Poortere, M Shayegan and R Winkler, Phys. Rev. B67, 241309(R) (2003)

[14] K Vakili, Y P Shkolnikov, E Tutuc, E P De Poortere and M Shayegan, Phys. Rev. Lett. 92, 226401 (2004)

[15] S Yarlagadda and G F Giuliani, Phys. Rev. B49, 7887 (1994); 61, 12556 (2000) C S Ting, T K Lee and J J Quinn, Phys. Rev. Lett. 34, 870 (1975)

[16] H M Böhm and K Schörkhuber, J. Phys.: Condens. Matter 12, 2007 (2000)

[17] Y Zhang and S Das Sarma, Phys. Rev. B71, 045322 (2005) S Das Sarma, V M Galitski and Y Zhang, Phys. Rev. B69, 125334 (2004)

[18] R Asgari, B Davoudi, M Polini, G F Giuliani, M P Tosi and G Vignale, Phys. Rev. B71, 045323 (2005)

[19] R Asgari, B Davoudi and B Tanatar, Solid State Commun. 130, 13 (2004)

[20] S De Palo, M Botti, S Moroni and G Senatore, Phys. Rev. Lett. 94, 226405 (2005)

[21] M W C Dharma-wardana, Phys. Rev. B72, 125339 (2005); Solid State Commun. 136, 76 (2005)

[22] L J Lantto and P J Siemens, Nucl. Phys. A317, 55 (1979) L J Lantto, Phys. Rev. B36, 5160 (1987)

[23] J G Zabolitzky, Phys. Rev. B22, 2353 (1980)

[24] E Krotscheck and M Saarela, Phys. Rep. 232, 1 (1993)

[25] T Chakraborty, Phys. Rev. B25, 3177 (1982); 26, 6131 (1982) T Chakraborty, A Kallio, L J Lantto and P Pietiläinen, Phys. Rev. B27, 3061 (1983)

[26] C A Kukkonen and A W Overhauser, Phys. Rev. B20, 550 (1979)

[27] R Asgari, A L Subaşı, A A Sabouri-Dodaran and B Tanatar, Phys. Rev. B74, 155319 (2006)

[28] B Davoudi, R Asgari, M Polini and M P Tosi, Phys. Rev. B68, 155112 (2003)

[29] A Kallio and J Piilo, Phys. Rev. Lett. 77, 4237 (1996)

[30] R Asgari and B Tanatar, Phys. Rev. B74, 075301 (2006)

[31] C Attaccalite, S Moroni, P Gori-Giorgi and G B Bachelet, Phys. Rev. Lett. 88, 256601 (2002) 